\title{
A simple interpretation of global trends in the lowest levels of $p$ - and sd-shell nuclei
}

\author{
G. Lévai ${ }^{1, a}$ and P.O. Hess ${ }^{2}$ \\ 1 Institute of Nuclear Research of the Hungarian Academy of Sciences (ATOMKI), Pf. 51 H-4001 Debrecen, Hungary \\ 2 Instituto de Ciencias Nucleares, UNAM, Circuito Exterior, C.U., A.P. 70-543, 04510 México D.F., Mexico
}

Received: 2 July 2005 /

Published online: 23 March 2006 - (c) Società Italiana di Fisica / Springer-Verlag 2006

\begin{abstract}
A simple approach is presented to estimate the position of the first opposite-parity state for pand sd-shell nuclei. The approach is based on the assumption that the quadrupole-quadrupole interaction determines the energy of the lowest-lying states, and they are a mixture of a few leading $S U(3)$ irreps even in the presence of further symmetry-breaking interactions. The quadrupole-quadrupole interaction together with the indirect effect of the Pauli exclusion principle will then lead to a rich structure in the trends of observables along $Z, N$ and $A$ chains. A comparison with the experimental data is given, with the carbon chain as illustrative example. The findings suggest that the changing shell structure near the neutron drip line might be explained by the appearance of low-lying highly deformed $2 \hbar \omega$ states.
\end{abstract}

PACS. 21.60.Fw Models based on group theory - 21.10.Hw Spin, parity and isobaric spin

\section{Introduction}

With the evolution of nuclear physics increasingly complex models have been developed in order to describe the structure of the nucleus, this many-body system of nucleons interacting via complicated forces. These models are considered successful if they are able to account for a wide variety of observables with reasonable accuracy. In order to fulfill these requirements it is usually necessary to use numerous parameters and basic assumptions when the models are constructed. Even then many models can be used only in a limited domain of nuclei, and even there their performance is considered really successful only for specific examples. At the same time the complicated machinery of these models often obscures the basic physical picture behind the phenomena they describe.

In light of this one often finds simple approaches, including even some oversimplified ones rather useful to gain insight into nuclear structure and to obtain thumb rules for certain structural properties of nuclei. For example, in [1] a two-level model was introduced which helped to understand pairing properties and the onset of deformation in nuclei. In [2] a simple picture was used to predict shell inversion in ${ }^{11} \mathrm{Be}$, while in [3] the structure of nuclei is looked upon from the simplest angle and trends in structure can be understood via elementary considerations. Such simple models can be as useful as very sophisticated ones, because they allow for a transparent descrip-

\footnotetext{
a e-mail: levai@atomki.hu
}

tion of gross structures and shed some light onto usually complicated situations.

In this contribution we present a schematic but systematic approach designed to account for some elementary observables of p- and sd-shell nuclei in a consistent way. These are the excitation energies of the lowest positiveand negative-parity states with special attention to the position of the first state with parity opposite to that of the ground state. This approach rests on the assumption that an extremely simplified (one-parameter) Hamiltonian dominated by the quadrupole-quadrupole interaction and observing the Pauli principle is sufficient to trace qualitatively the position of these states. It is assumed that these states originate from the lowest (i.e. the $0 \hbar \omega, 1 \hbar \omega$ and $2 \hbar \omega)$ shells and have dominant contribution only from a few leading $S U(3)$ basis states of the Elliott model [4]. It then follows that even if the $S U(3)$ symmetry is broken, the relative energies of these states are not influenced significantly by the mixing.

The rationale of this simple approach is that focusing only on certain basic observables of a large number of nuclei (from ${ }^{4} \mathrm{He}$ to ${ }^{40} \mathrm{Ca}$ ) can be at least as useful as more sophisticated models that describe many levels of one (or a few) nucleus. Despite its simplicity, the present approach takes into account the most important factors in this region: the quadrupole-quadrupole interaction that generates deformation and the Pauli principle that selects the allowed $(S U(3))$ configurations. The interplay of these two key elements then generates characteristic structural changes in the trend of the fundamental observables 
Table 1. Leading $S U(3)$ representations associated with $n$ identical nucleons (protons or neutrons) occupying the $i$-th shell, and the corresponding permutational symmetry $[f]$. For configurations with $n>6$ on the sd shell the conjugate representation belonging to $12-n$ has to be taken. Only configurations appearing up to $2 \hbar \omega$ excitations are displayed.

\begin{tabular}{|c|c|c|c|c|c|c|c|c|c|}
\hline$i$ & Shell & $\begin{array}{c}n \\
{[f]}\end{array}$ & $\begin{array}{c}0 \\
{[0]}\end{array}$ & $\begin{array}{c}1 \\
{[1]}\end{array}$ & $\begin{array}{c}2 \\
{[11]}\end{array}$ & $\begin{array}{c}3 \\
{[21]}\end{array}$ & $\begin{array}{c}4 \\
{[22]}\end{array}$ & $\begin{array}{c}5 \\
{[32]}\end{array}$ & $\begin{array}{c}6 \\
{[33]}\end{array}$ \\
\hline 1 & $\mathrm{~s}$ & & $(0,0)$ & $(0,0)$ & $(0,0)$ & & & & \\
\hline 2 & $\mathrm{p}$ & & $(0,0)$ & $(1,0)$ & $(2,0)$ & $(1,1)$ & $(0,2)$ & $(0,1)$ & $(0,0)$ \\
\hline 3 & sd & & $(0,0)$ & $(2,0)$ & $(4,0)$ & $(4,1)$ & $(4,2)$ & $(5,1)$ & $(6,0),(0,6)$ \\
\hline 4 & $\mathrm{pf}$ & & $(0,0)$ & $(3,0)$ & $(6,0)$ & & & & \\
\hline 5 & $\operatorname{sdg}$ & & $(0,0)$ & $(4,0)$ & & & & & \\
\hline
\end{tabular}

we investigate, and does so without incorporating further model assumptions concerning e.g. the deformation of nuclei, which is an important input for alternative models. Another advantage of the $S U(3)$ scheme is that it is relatively easy to pin down regions where the basic assumptions are expected to break down, and where the results thus have to be accepted with caution. It is also possible to estimate the importance of the neglected interactions (pairing, spin-orbit, etc.)

The aim of this study is not the description of individual nuclei, rather to search for trends in the mass, neutron and proton number, which might indicate structural changes. This can be instructive for experiments targeting unexplored regions of the nuclide chart.

\section{The approach}

In this section we present the basic assumptions of our approach and discuss their importance together with their validity in various domains of p- and sd-shell nuclei [5]. Assumption 1. The lowest-lying states can reasonably be described in terms of the harmonic oscillator shell model of Elliott [4] in the sense that they have dominant contribution only from a few leading $S U(3)$ basis states.

The validity of the Elliott model has been proven for many light nuclei, which means that the physical states can be described reasonably well in terms of the $S U(3)$ basis states of the model. This implies that the $S U(3)$ symmetry is largely respected by the interaction terms considered in the Hamiltonian. The most important term in the Elliott Hamiltonian is the $\mathbf{Q} \cdot \mathbf{Q}$ quadrupole-quadrupole interaction, which is closely related to the second-order Casimir invariant $\mathbf{C}_{2}$ of the $S U(3)$ group. In fact, in the best examples of the Elliott model $\left({ }^{20} \mathrm{Ne},{ }^{24} \mathrm{Mg}\right.$, etc. $)$ the physical states are almost pure $S U(3)$ states belonging to the leading $S U(3)$ irreducible representation (irrep) $(\lambda, \mu)$, i.e. the one for which $\mathbf{C}_{2}$ has maximal eigenvalue $C_{2}(\lambda, \mu)=\lambda^{2}+\mu^{2}+\lambda \mu+3 \lambda+3 \mu$. These leading $S U(3)$ states are also the most deformed ones. While the overall magnitude of $\lambda$ and $\mu$ determine the magnitude of the deformation, their relative size is indicative for the nature of the deformation. In particular, irreps with large $\lambda$ and small $\mu$ represent prolate deformation, while the reverse situation corresponds to oblate shape. When neither numbers are small compared to the other, a triaxial shape occurs.

Obviously, in most cases the $S U(3)$ symmetry is broken, nevertheless, it was found that even when this happens, only $S U(3)$ irreps with similar $C_{2}(\lambda, \mu)$ get mixed [6], so the average expectation value of the Casimir invariant $\mathbf{C}_{2}$ is close to the expectation value for the leading $S U(3)$ irrep. If this mechanism holds not only for the $0 \hbar \omega$ states, but also for the lowest-lying $1 \hbar \omega$ and $2 \hbar \omega$ states, then the energy differences of the opposite-parity levels are moderately sensitive to the actual symmetry breaking.

This scenario is a reasonable approximation in midshell situations, but it might fail close to shell closures, where there are fewer $S U(3)$ irreps and even these have different $C_{2}(\lambda, \mu)$ expectation value. Furthermore, the breakdown of the $S U(3)$ symmetry is also stronger near the shell closures, i.e. where $N$ and $Z$ are close to 2,8 and 20 .

In general the construction of the full $S U(3)$ model space is a difficult task, however, the leading $S U(3)$ irreps can be identified relatively easily. Due to the total antisymmetry of the nuclear wave function, the spatial and the spin-isospin structure of the nucleus is correlated. In practical terms this means that the maximal spatial symmetry (exhibiting itself in maximal deformation) comes with maximal antisymmetry in the spin-isospin sector, so in the lowest-lying configurations the protons and neutrons tend to pair off separately. There is thus a relatively simple recipe which we can use to determine the $S U(3)$ character of the states in question by extracting the $S U(3)$ content of the proton and neutron configurations on each shell (s, p, sd, pf and sdg, the latter two only in excited configurations), and combining them to obtain the largest possible $(\lambda, \mu)$ irrep. Table 1 lists the $(\lambda, \mu)$ quantum numbers associated with proton and neutron configurations possessing maximal symmetry in the spatial sector [4]. We list all configurations on the $\mathrm{s}$ and $\mathrm{p}$ shells, while for the sd shell we present the $(\lambda, \mu)$ irreps up to particle number $n=6$, which corresponds to the middle of the shell: for $n>6$ the appropriate $S U(3)$ irreps are obtained as the conjugates $(\mu, \lambda)$ of the $S U(3)$ irreps belonging to $12-n<6$ particles. In table 1 the pf and sdg shells appear with configurations consisting of up to 2 and 1 particles, respectively, because only such systems 
Table 2. Possible nucleon configurations and leading $S U(3)$ representations for 0,1 and $2 \hbar \omega$ in the proton and neutron sector for ${ }^{16} \mathrm{C}$.

\begin{tabular}{lllll}
\hline \multicolumn{4}{c}{ Protons } & \multicolumn{2}{c}{ Neutrons } \\
\hline $0 \hbar \omega$ & $\mathrm{s}^{2} \mathrm{p}^{4}$ & $(0,2)$ & $\mathrm{s}^{2} \mathrm{p}^{6} \mathrm{sd}^{2}$ & $(4,0)$ \\
$1 \hbar \omega$ & $\mathrm{s}^{2} \mathrm{p}^{3} \mathrm{sd}^{1}$ & $(3,1)$ & $\mathrm{s}^{2} \mathrm{p}^{6} \mathrm{sd}^{1} \mathrm{pf}^{1}$ & $(5,0)$ \\
& $\mathrm{s}^{1} \mathrm{p}^{5}$ & $(0,1)$ & $\mathrm{s}^{2} \mathrm{p}^{5} \mathrm{sd}^{3}$ & $(4,2)$ \\
$2 \hbar \omega$ & $\mathrm{s}^{2} \mathrm{p}^{3} \mathrm{pf}^{1}$ & $(4,1)$ & $\mathrm{s}^{2} \mathrm{p}^{6} \mathrm{sd}^{1} \mathrm{sdg}^{1}$ & $(6,0)$ \\
& $\mathrm{s}^{2} \mathrm{p}^{2} \mathrm{sd}^{2}$ & $(6,0)$ & $\mathrm{s}^{2} \mathrm{p}^{6} \mathrm{pf}^{2}$ & $(6,0)$ \\
& $\mathrm{s}^{1} \mathrm{p}^{4} \mathrm{sd}^{1}$ & $(2,2)$ & $\mathrm{s}^{2} \mathrm{p}^{5} \mathrm{sd}^{2} \mathrm{pf}^{1}$ & $(7,1)$ \\
& $\mathrm{s}^{0} \mathrm{p}^{6}$ & $(0,0)$ & $\mathrm{s}^{2} \mathrm{p}^{4} \mathrm{sd}^{4}$ & $(4,4)$ \\
& & & $\mathrm{s}^{1} \mathrm{p}^{6} \mathrm{sd}^{3}$ & $(4,1)$ \\
\hline
\end{tabular}

can appear when we determine $1 \hbar \omega$ and $2 \hbar \omega$ excitations of sd-shell nuclei. Table 1 also contains the permutational symmetry $[f]$ associated with the proton and neutron configurations on each major shell, and it is a straightforward task to construct the possible permutational symmetries of both nucleon types and of the whole nuclear state.

In order to determine the leading $S U(3)$ irrep $(\lambda, \mu)$ for configurations without major shell excitation $(0 \hbar \omega)$ we simply have to take the $\left(\lambda_{\pi}^{i}, \mu_{\pi}^{i}\right)$ and $\left(\lambda_{\nu}^{i}, \mu_{\nu}^{i}\right)$ irreps obtained for protons and neutrons from the $i$-th shell (s, $\mathrm{p}$, sd, pf, sdg for $i=1,2,3,4,5$, respectively), and combine them to get $\lambda=\sum_{i=1}^{5}\left(\lambda_{\pi}^{i}+\lambda_{\nu}^{i}\right), \mu=\sum_{i=1}^{5}\left(\mu_{\pi}^{i}+\mu_{\nu}^{i}\right)$. We illustrate this procedure with the example of the ${ }^{16} \mathrm{C}$ nucleus consisting of $Z=6$ protons and $N=10$ neutrons. Table 2 displays the possible proton and neutron configurations up to two shell excitation quanta, together with the corresponding leading $\left(\lambda_{\pi}^{i}, \mu_{\pi}^{i}\right)$ and $\left(\lambda_{\nu}^{i}, \mu_{\nu}^{i}\right) S U(3)$ irreps. The leading $0 \hbar \omega$ is then found to be $(4,2)$.

As can be seen from table 2, there are several configurations for $1 \hbar \omega$ depending on whether we excite one nucleon from the valence shell to the next highest shell or from the shell below to the valence shell. We have to calculate the leading irrep from each of these following the recipe given above. The leading $S U(3)$ irrep is found to be $(7,1)$, which originates from the $(3,1)$ proton and $(4,0)$ neutron configuration. It also has to be mentioned that in order to get rid of spurious states, the center-of-mass motion has to be subtracted. In the harmonic-oscillator picture this is done very easily: the $S U(3)$ irreps of the $0 \hbar \omega$ model space have to be multiplied by the $(1,0) S U(3)$ irrep representing one excitation quantum in the c.m. motion, and the resulting $(\lambda, \mu)$ states have to be subtracted from the $1 \hbar \omega$ model space obtained before [7]. The largest $(\lambda, \mu)$ irrep with multiplicity larger than 0 will then be the leading one for $1 \hbar \omega$. It is obvious from table 2 that the $(7,1)$ irrep is not redundant. The $2 \hbar \omega$ space can be constructed in a similar way: proton and neutron configurations with altogether two shell excitations have to be considered. Then the leading $S U(3)$ irrep is found to be $(10,0)$, which originates from the $2 \hbar \omega(6,0)$ proton and the $0 \hbar \omega(4,0)$ neutron configuration.

It has to be mentioned that in certain special situations the simple recipe outlined above might not produce automatically the leading $S U(3)$ state. This is the case in the middle of the sd shell, when 6 identical nucleons can yield the $(6,0)$ and also the $(0,6) S U(3)$ state, as can be seen from table 1 . In these ambiguous situations further assumptions have to be made in order to select the configuration that leads to the leading $S U(3)$ irrep of the total wave function. The general rule is that the contribution of the other type of nucleons, as well as that of the same type of nucleons from the $\mathrm{p}$ shell have to be taken into consideration: the leading $S U(3)$ irrep is obtained if $\lambda$ or $\mu$ reaches the possible maximal value. This corresponds to a kind of "polarization" effect in the sense that the two types of nucleons attempt to realize similar kind of deformation, i.e. prolate or oblate. In fact, this effect can appear in $\mathrm{sd}^{5}$ and $\mathrm{sd}^{7}$ configurations too, to which the simple rule would assign $(5,1)$ and $(1,5)$ as in table 1 , while the construction of the true leading $S U(3)$ state might require the secondary $(2,4)$ and $(4,2)$ states, with opposite kind of deformation. Assumption 2. The simplified Hamiltonian

$$
H=\hbar \omega \mathbf{N}-\chi \mathbf{C}_{2}(\lambda, \mu)
$$

is sufficient to account for the excitation energy of the lowest few levels.

Here the first term accounts for major shell excitations $(\mathbf{N}=0,1,2)$, while $\mathbf{C}_{2}$ is the second-order Casimir operator of $S U(3)$ mentioned earlier. Since the states are supposed to be composed of several $S U(3)$ basis states the expectation value of $\mathbf{C}_{2}$ should be the corresponding average of the $C_{2}(\lambda, \mu)$ eigenvalues. However, based on Assumption 1 we replace this average with the eigenvalue belonging to the leading $S U(3)$ state, which can be determined using the mechanism presented previously. The two coupling constants appearing in (1) are known to be parametrized in terms of $A$ only. In particular, we can take the formula

$$
\hbar \omega=45 A^{-1 / 3}-25 A^{-2 / 3},
$$

(in units of $\mathrm{MeV}$ ), which was deduced from the systematic behaviour for light nuclei near the valley of stability [8]. This value might thus change when the neutron drip line is approached: due to loosely bound neutrons the average $\hbar \omega$ might be lowered. For $\chi$ we take

$$
\chi=a A^{-\frac{5}{3}}
$$

which is a generally accepted parametrization [9]. We adjusted $a$, the only parameter appearing in our approach to the $\chi$ value calculated for 100 nuclei using the expression (1) and the experimental energy of the first oppositeparity level [5]. The trend of $\chi$ and its parametrization in terms of (3) is displayed in fig. 1 using the adjusted $a=30$ $\mathrm{MeV}$ value. The deviation for low $A$ might be partly due to the underestimation of $\hbar \omega$ : this is indicated by the fact that the adjusted $\chi$ is negative in a number of cases, which is the consequence of the fact that $\hbar \omega$ is lower than the energy of the lowest-lying opposite-parity state. The increasing trend of the data points towards $A=40$ and the maximum near $A=16$ might be an indirect effect of the shell closure too, which may lead to deviations from the smooth behaviour of $\hbar \omega$ in (2) and/or $\chi$ in (3). This might 


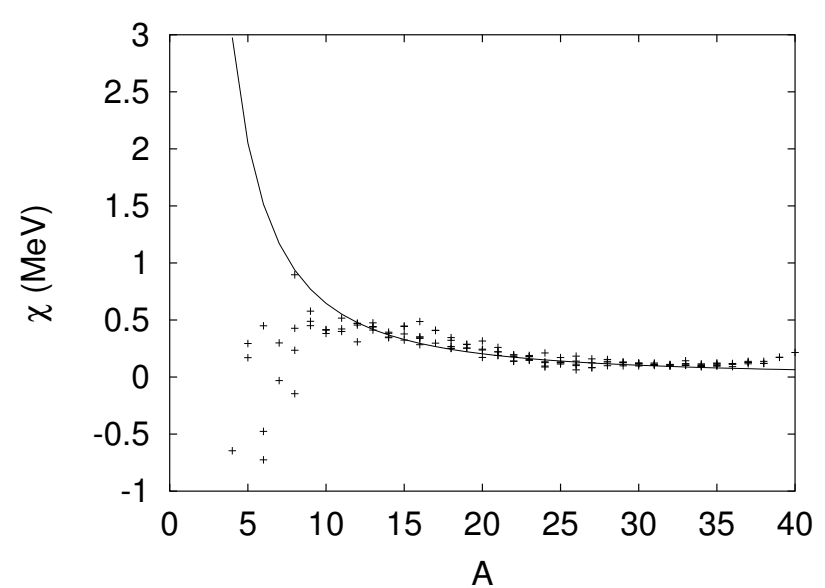

Fig. 1. The parameter $\chi$ as a function of the mass number $A$ (full line). The parametrization $a A^{-\frac{5}{3}}$ was chosen with $a=$ $30 \mathrm{MeV}$. Crosses indicate $\chi$ values calculated back from (1) using the experimental energy of the lowest opposite-parity level.

be related to the observation made before, i.e. that the validity of the $S U(3)$ scheme might break down near shell closures, where the importance of the $\mathbf{Q} \cdot \mathbf{Q}$ interaction is expected to be reduced. In practical terms this means that the $S U(3)$ breaking interactions (e.g., pairing) might mix further $S U(3)$ states with the leading ones, so the replacement of $\left\langle C_{2}(\lambda, \mu)\right\rangle$ with the $C_{2}(\lambda, \mu)$ eigenvalue of the leading $S U(3)$ irrep might not be a good approximation in this case. In fact, this effect could be compensated by taking larger $\chi$ values near the shell closures, which is exactly what fig. 1 indicates in these regions.

In a full scale spectroscopic study further terms should also be considered in (1), however, if we focus only on lowlying levels that have low values for the orbital angular momentum and spin, terms like the spin-orbit interaction, $\mathbf{L}^{2}, \mathbf{L} \cdot \mathbf{S}$ and spin-dependent forces are not expected to contribute significantly to the energy [5].

Before closing this section we return to the major assumptions of our approach, i.e. that in order to account for the general trends in the lowest-lying levels of nuclei in this region it is sufficient to incorporate the two most important factors, i.e. the quadrupole-quadrupole interaction that generates deformation and the Pauli principle that selects the allowed $(S U(3))$ configurations. The interplay of these two key elements then generates characteristic structural changes in the trend of the fundamental observables we investigate, and does so without incorporating further model assumptions concerning e.g. the deformation of nuclei, which is an important input for alternative models. Obviously, the breakdown of $S U(3)$ symmetry and thus the mixing of $S U(3)$ states has strong influence on further observables. This is the case, for example, with the electric quadrupole moment, because its expectation value is calculated from quantities that significantly differ from each other in their magnitude, and in addition, might also have different sign. Nevertheless, $Q$ can be calculated in a straightforward and parameter-free

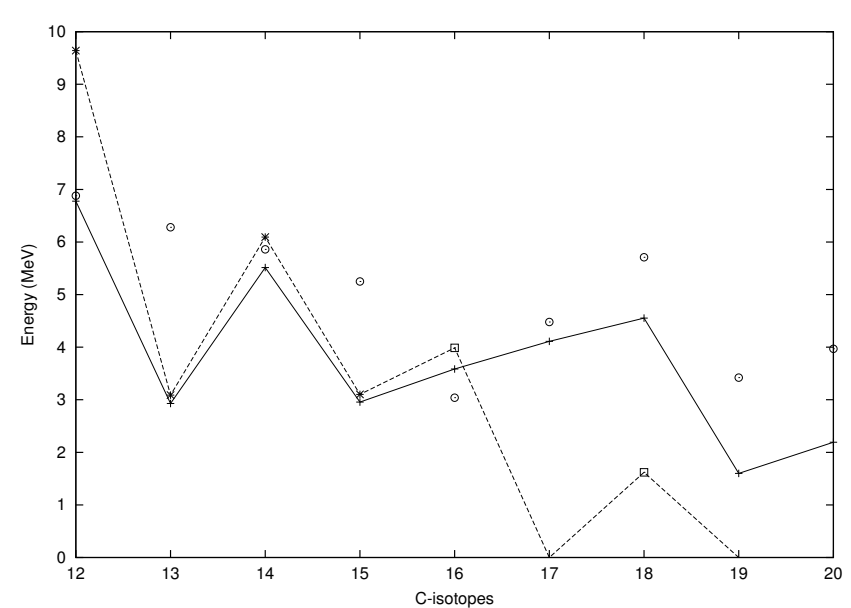

Fig. 2. The energy of the lowest opposite-parity $(1 \hbar \omega)$ level in the $\mathrm{C}$ isotopes, and the lowest expected $2 \hbar \omega$ level (open circles). Dashed and full lines connect experimental and theoretical points, respectively. Crosses indicate experimentally well-established opposite-parity states, while open boxes stand for lower limits in energy. The lack of symbol stands for missing data.

way for any $S U(3)$ state [5], and this can be helpful in determining the character of the nuclear states.

\section{Applications}

In [5] we presented the results concerning the position of the first opposite-parity state and compared it to experiment, as far as data were available. In total about 180 nuclei in the $\mathrm{p}$ and sd shell were considered and of those predictions were made for about 80 nuclei. In general, the agreement was satisfactory. The general trend was well reproduced, although not always in terms of absolute values. As illustration we present our results in tabular and graphical form in table 3 and fig. 2 for the carbon isotope chain. It is seen from fig. 2 that the rich structure of the experimental plot is excellently reproduced by the calculated one and not only in its trend, but also in magnitude. It is remarkable that the odd-even staggering structure is reproduced without any spin-dependent interactions, by considering only terms depending on the orbital structure of the nuclei. This odd-even staggering effect appeared in other isotope chains too, mainly with even value of $Z$ (Be, $\mathrm{Ne}, \mathrm{Mg}, \mathrm{Si}, \mathrm{S})$ [5], and apart from the $\mathrm{Mg}$ chain, even its magnitude was reproduced reasonably well. Another characteristic feature reproduced rather successfully was a bump near $N=8$ in the $\mathrm{N}, \mathrm{O}$ and $\mathrm{F}$ chain, which obviously originates from the shell closure effect.

Deviations from the experimental plot appeared in certain well-defined regions. This was the case for light isotopes $A \leq 10$, where the theoretical curve systematically fell behind the experimental one, leading even to shell inversion in some cases. As we have discussed before, this might be the consequence of the underestimation of $\hbar \omega$ in (2) in this region. We note that shell inversion (i.e. $E(1 \hbar \omega)<E(0 \hbar \omega))$ was reproduced for ${ }^{11}$ Be too, the only 
Table 3. Numerical values of $\hbar \omega$ and $\chi$ used in (1) to determine the energies of the lowest $1 \hbar \omega$ and $2 \hbar \omega$ states $\left(E_{\mathrm{Th}}(1 \hbar \omega)\right.$, $\left.E_{\mathrm{Th}}(2 \hbar \omega)\right)$ with the indicated $(\lambda, \mu)$ quantum numbers for the $\mathrm{C}$ isotopes, displayed together with experimental information for the ground and the lowest opposite-parity state.

\begin{tabular}{|c|c|c|c|c|c|c|c|c|c|c|}
\hline \multirow[t]{2}{*}{ Nucleus } & \multirow[t]{2}{*}{$\hbar \omega$} & \multirow[t]{2}{*}{$\chi$} & \multicolumn{3}{|c|}{$(\lambda, \mu)$} & \multirow[t]{2}{*}{$J_{\mathrm{g} . \mathrm{s} .}^{\pi}$} & \multicolumn{2}{|c|}{$1^{\text {st }}$ opp. p. state } & \multirow[t]{2}{*}{$E_{\mathrm{Th}}(1 \hbar \omega)$} & \multirow[t]{2}{*}{$E_{\mathrm{Th}}(2 \hbar \omega)$} \\
\hline & & & $0 \hbar \omega$ & $1 \hbar \omega$ & $2 \hbar \omega$ & & $E_{\operatorname{Exp}}$ & $J^{\pi}$ & & \\
\hline${ }^{12} \mathrm{C}$ & 14.89 & 0.477 & $(0,4)$ & $(3,3)$ & $(6,2)$ & $0^{+}$ & 9.641 & $3^{-}$ & 6.778 & 6.878 \\
\hline${ }^{13} \mathrm{C}$ & 14.62 & 0.417 & $(0,3)$ & $(2,4)$ & $(5,3)$ & $\frac{1}{2}^{-}$ & 3.089 & $\frac{1}{2}^{+}$ & 2.929 & 6.276 \\
\hline${ }^{14} \mathrm{C}$ & 14.37 & 0.369 & $(0,2)$ & $(2,3)$ & $(4,4)$ & $0^{+}$ & 6.093 & $1^{-}$ & 5.514 & 5.863 \\
\hline${ }^{15} \mathrm{C}$ & 14.14 & 0.329 & $(2,2)$ & $(4,3)$ & $(7,2)$ & $\frac{1}{2}^{+}$ & 3.103 & $\frac{1}{2}^{-}$ & 2.956 & 5.254 \\
\hline${ }^{16} \mathrm{C}$ & 13.92 & 0.295 & $(4,2)$ & $(7,1)$ & $(10,0)$ & $0^{+}$ & $\geq 3.986$ & 2 & 3.586 & 3.037 \\
\hline${ }^{17} \mathrm{C}$ & 13.72 & 0.267 & $(4,3)$ & $(7,2)$ & $(10,1)$ & n.d. & $n . d$. & n.d. & 4.111 & 4.485 \\
\hline${ }^{18} \mathrm{C}$ & 13.53 & 0.243 & $(4,4)$ & $(7,3)$ & $(10,2)$ & $\left(0^{+}\right)$ & $>1.62$ & n.d. & 4.552 & 5.707 \\
\hline${ }^{19} \mathrm{C}$ & 13.35 & 0.222 & $(5,3)$ & $(7,4)$ & $(10,3)$ & n.d. & $n . d$. & n.d. & 1.600 & 3.422 \\
\hline${ }^{20} \mathrm{C}$ & 13.18 & 0.204 & $(0,8)$ & $(8,3)$ & $(10,4)$ & n.d. & n.d. & n.d. & 4.635 & 3.976 \\
\hline
\end{tabular}

known isotope for which the parity of the ground state differs from that corresponding to a $0 \hbar \omega$ configuration. Although this nucleus is close to the $A \leq 10$ region, fig. 1 demonstrates that in this case the low $E_{\mathrm{Th}}(1 \hbar \omega)$ value is not due to the underestimated $\hbar \omega$ : the cross corresponding to ${ }^{11}$ Be lies almost precisely on the $\chi$ curve calculated from (3).

One more systematic deviation appears near $A=40$, where the $E_{\mathrm{Th}}(1 \hbar \omega)$ falls behind the energy of the first opposite-parity level. This, again, is obviously due to the shell closure effect which influences the results through an underestimated $\chi$ value. This can also be traced down on fig. 1.

The trends described here can be understood from table 3, where the $S U(3)$ states $(\lambda, \mu)$ are displayed for the leading 0,1 and $2 \hbar \omega$ states. It is seen that proceeding along the isotope chain $\lambda$ and $\mu$ rarely change with more than one unit as the shells are filled up with more and more neutrons. There are, however, some discontinuities for 1 and $2 \hbar \omega, e . g$. at ${ }^{16} \mathrm{C}$. This is due to the fact that particles excited to higher shells typically contribute to the whole system with $S U(3)$ configurations corresponding to prolate deformation (see table 2 ). This shows up in a low $E_{\mathrm{Th}}(2 \hbar \omega)$ level too, as can be seen in fig. 2 . It is a general trend in other isotopes too that the $0 \hbar \omega$ state can be prolate, oblate or triaxial type, while the excited states tend to proceed towards the prolate direction with relatively large deformation.

We mention here that the ${ }^{16} \mathrm{C}$ nucleus is known to exhibit some unusual features. It was found, for example, that there is an extremely weak electric quadrupole transition from the first $2^{+}$state at $E_{x}=1.766 \mathrm{MeV}$ to the ground state [10], furthermore, the observations were compatible with a large deformation, especially for protons. In our scheme the leading $0 \hbar \omega$ and $2 \hbar \omega$ states belong to the $(\lambda, \mu)=(4,2)$ and $(10,0) S U(3)$ irreps, which certainly have rather different structure, leading to a strong hindrance in the electromagnetic transitions between them. The $2 \hbar \omega(10,0)$ states also have strongly prolate nature; in particular, they are constructed from an $\mathrm{s}^{2}, \mathrm{p}^{2}, \mathrm{sd}^{2}$ proton structure, having $(6,0) S U(3)$ character, and an $\mathrm{s}^{2}$, $\mathrm{p}^{6}, \mathrm{sd}^{2}$ neutron structure, having $(4,0)$ (see table 2$)$. This is clearly compatible with the experimental findings. The simple scheme yields the energy of the lowest $(10,0)$ state at $E_{x}=3.04 \mathrm{MeV}$, which is also rather close to the experimental energy of the $2_{1}^{+}$state. In summary, we assume that even if the $S U(3)$ symmetry is broken and the states do not have pure $S U(3)$ character, the ground state of ${ }^{16} \mathrm{C}$ is dominated by $0 \hbar \omega$, while the $2_{1}^{+}$state has dominantly $2 \hbar \omega$ structure. This also means that there should be a $0_{2}^{+}$state with dominantly $2 \hbar \omega$, and a $2_{2}^{+}$state with dominantly $0 \hbar \omega$ character in the vicinity of these levels. The states at $E_{x}=3.027 \mathrm{MeV}$ and $3.987 \mathrm{MeV}$ might be candidates for these.

The example of the ${ }^{16} \mathrm{C}$ nucleus shows that discontinuities in some basic observables (e.g. quadrupole moment, $B(E 2)$, deformation) in the ground-state region of nuclei can be the result of the presence of highly deformed lowlying $2 \hbar \omega$ states. This mechanism eventually leads to the smearing out of the energy gap and the apparent disappearance of the shell structure. This scenario can also occur near the closure of the $N=20$ (or $Z=20$ ) shell, since the $0 \hbar \omega$ configuration has small deformation there, while exciting nucleons to the pf and sdg shells may change the deformation to the strongly prolate direction.

There is one more interesting finding found in some nuclei that can be illustrated with the example of a carbon isotope. In particular, there is a strong discontinuity in the $0 \hbar \omega(\lambda, \mu)$ state at ${ }^{20} \mathrm{C}$ : here the protons correspond to the usual $\mathrm{p}^{2}(0,2)$ configuration, while the neutrons are assigned to $\operatorname{sd}^{6}(0,6)$ or $(6,0)$ (see table 1 ). Due to the "polarization" effect discussed previously the lowest energy arises if the $(0,6)$ neutron state is taken, leading to the overall $(0,8)$ state (see table 3$)$. The $\mathrm{sd}^{6}$ configurations typically appear at nuclei with $N, Z=14$, and can lead to underestimated $E_{\mathrm{Th}}(1 \hbar \omega)$ or $E_{\mathrm{Th}}(2 \hbar \omega)$ energies in a handful of isotopes near the middle of the sd shell [5].

Before closing this section, we mention that calculations for the quadrupole moments for about sixty nuclei with known $Q_{\operatorname{Exp}}$ or $\left|Q_{\operatorname{Exp}}\right|$ showed that the picture in which a few dominant $S U(3)$ irreps get mixed in the lowest-lying states is consistent with the experimental data [5]. While $Q_{\text {Th }}$ calculated using the leading $0 \hbar \omega S U(3)$ state agrees remarkably well with $Q_{\operatorname{Exp}}$ for nuclei that are known have pure $S U(3)$ character in their ground state $\left({ }^{20} \mathrm{Ne},{ }^{24} \mathrm{Mg},{ }^{19} \mathrm{~F}\right.$, etc. $)$, it represents an up- 
per limit in the case of most other nuclei, indicating that some other leading $S U(3)$ states mix with the leading one significantly. In some cases $Q_{\operatorname{Exp}}$ was also close to $Q_{\mathrm{Th}}$ calculated with the leading $2 \hbar \omega$ state indicating that such states might play important role even in the ground-state region.

\section{Conclusions}

We presented the basic ideas of a simple thumb rule approach designed to estimate the position of the first opposite-parity $(1 \hbar \omega)$ state and the first $2 \hbar \omega$ state in light nuclei. The full details of the method and the specific results concerning about 180 nuclei are given in [5], while here we discussed in more detail only the $\mathrm{C}$ isotope chain to illustrate the main findings. The method is specially directed to experimental physicists and they can use it to have a first estimate where to look for low lying opposite parity states or, even more difficult, where to look at shell inversion without the change in parity, i.e. shape isomeric states, which is related to the inversion with the $2 \hbar \omega$ shell.

The approach rests on two basic assumptions: 1) the lowest-lying states are composed only of a few leading $(\lambda, \mu) S U(3)$ irreps having comparable $C_{2}(\lambda, \mu) S U(3)$ eigenvalue; and 2) the terms other than the harmonic and quadrupole-quadrupole interaction do not contribute significantly to the energy. The approach contains only one adjustable parameter (the coupling constant of the quadrupole-quadrupole interaction), furthermore, the deformation of the nuclei follows directly from the calculations, rather than being an input parameter.

The simple rules outlined above imply that the joint action of the quadrupole-quadrupole interaction and the Pauli principle is able to reproduce the rich structure manifested in the energy of the first opposite-parity state, apart from some well-defined regions. The basic assumptions also guarantee that even if the $S U(3)$ symmetry is broken, this does not influence significantly the relative energy of the lowest 0,1 and $2 \hbar \omega$ levels. Furthermore, although other observables are more sensitive to the mixing of $S U(3)$ states, some of these, e.g. the quadrupole moment can be indicative of the nature (e.g. deformation) of the states.
With the example of the $\mathrm{C}$ isotopes and ${ }^{16} \mathrm{C}$ in particular, we demonstrated that some unusual findings usually attributed to the disappearance of the shell structure can be explained by our procedure. Though at $0 \hbar \omega$ the $S U(3)$ irrep is small near a closed shell or $(0,0)$ at a closed shell, at $n \hbar \omega(n>0)$ the corresponding $S U(3)$ irrep can represent large deformation at low energy. This result does suggest that the mean field description of nuclei near the neutron drip line can still be valid and the disappearance of the shell structure is only apparent. This effect is the result of the Pauli exclusion principle which allows only small $S U(3)$ irreps for nuclei near a closed shell but results in large $S U(3)$ irreps, and thus a large quadrupole moment and deformation for $2 \hbar \omega$ excitations. This is also related to the fact that in light nuclei small changes in the size of the $S U(3)$ irreps imply large changes in deformation, which is a less drastic effect for heavier nuclei.

This work was supported by the CONACyT-MTA and CSICMTA exchange programs, CONACyT, DGAPA (Grant No. IN119002) and by the OTKA (Grant No. T37502).

\section{References}

1. H.J. Lipkin, N. Meschkov, S. Glick, Nucl. Phys. A 62, 118 (1965).

2. I. Talmi, I. Unna, Phys. Rev. Lett. 4, 469 (1960).

3. R.F. Casten, Nuclear Structure from a Simple Perspective (Oxford University Press, Oxford, 1990).

4. J.P. Elliott, Proc. R. Soc. London, Ser. A 245, 128; 562 (1958).

5. P.O. Hess, G. Lévai, Int. J. Mod. Phys. E 14, 845 (2005).

6. J.G. Hirsch, P.O. Hess, L. Hernández, C. Vargas, T. Beuschel, J.P. Draayer, Rev. Mex. Fís. 45, (S2) 86 (1999); C.E. Vargas, J.G. Hirsch, J.P. Draayer, Nucl. Phys. A 691, 409 (2001); 697, 655 (2002).

7. J.P. Elliott, T.H.K. Skyrme, Proc. R. Soc. London, Ser. A 232, 561 (1955); D.M. Brink, G.F. Nash, Nucl. Phys. A 40, 608 (1963); K.T. Hecht, Nucl. Phys. A 170, 34 (1971).

8. J. Blomqvist, A. Molinari, Nucl. Phys. A 106, 545 (1968).

9. M. Dufour, A.P. Zuker, Phys. Rev. C 54, 1641 (1996).

10. N. Imai et al., Phys. Rev. Lett. 92, 62501 (2004). 